\title{
Theorems on the core of an economy with infinitely many commodities and consumers
}

\author{
Özgür Evren ${ }^{\mathrm{a}, *}$, Farhad Hüsseinov ${ }^{\mathrm{b}}$ \\ a Department of Economics, New York University, 19th West 4th Street, New York, NY 10012, USA \\ ${ }^{\mathrm{b}}$ Department of Economics, Bilkent University, Bilkent 06800, Ankara, Turkey
}

Received 28 September 2006; received in revised form 29 January 2008; accepted 31 January 2008

Available online 9 February 2008

\begin{abstract}
It is known that the classical theorems of Grodal [Grodal, B., 1972. A second remark on the core of an atomless economy. Econometrica 40, 581-583] and Schmeidler [Schmeidler, D., 1972. A remark on the core of an atomless economy. Econometrica $40,579-580$ ] on the veto power of small coalitions in finite dimensional, atomless economies can be extended (with some minor modifications) to include the case of countably many commodities. This paper presents a further extension of these results to include the case of uncountably many commodities. We also extend Vind's [Vind, K., 1972. A third remark on the core of an atomless economy. Econometrica 40, 585-586] classical theorem on the veto power of big coalitions in finite dimensional, atomless economies to include the case of an arbitrary number of commodities. In another result, we show that in the coalitional economy defined by an atomless individualistic model, core-Walras equivalence holds even if the commodity space is non-separable. The above-mentioned results are also valid for a differential information economy with a finite state space. We also extend Kannai's [Kannai, Y., 1970. Continuity properties of the core of a market. Econometrica 38, 791-815] theorem on the continuity of the core of a finite dimensional, large economy to include the case of an arbitrary number of commodities. All of our results are applications of a lemma, that we prove here, about the set of aggregate alternatives available to a coalition. Throughout the paper, the commodity space is assumed to be an ordered Banach space which has an interior point in its positive cone.
\end{abstract}

(C) 2008 Elsevier B.V. All rights reserved.

JEL classification: C62; C71; D41; D51; D82

Keywords: Small coalitions; Core; Strong core; Private core; Walrasian equilibrium; Radner equilibrium; Stability; Continuity; Differential information; Non-separable commodity space

\section{Introduction}

In this paper, we show that if a coalition blocks an allocation, that coalition can in fact block that allocation by disposing a strictly positive amount of its resources, provided that all of its subcoalitions have strictly positive endowments. We then use this lemma to prove several useful facts about the core of an economy with infinitely many commodities and consumers. The assumptions that we use in our main results are satisfied by the models considered in the equilibrium existence result of Khan and Yannelis (1991), the core non-emptiness result of Podczeck (2003),

\footnotetext{
* Corresponding author. Fax: +1 2129954186.

E-mail addresses: oe240@nyu.edu (Ö. Evren), farhad@bilkent.edu.tr (F. Hüsseinov).
} 
the core-Walras equivalence result of Podczeck (2003), and ignoring some minor points, the core-Walras equivalence result of Rustichini and Yannelis (1991, Theorem 4.1). Throughout the paper, we assume that there is no production sector and that the commodity space is an ordered Banach space which has an interior point in its positive cone. The aggregation of commodity bundles will be formalized via the Bochner integral.

Assuming that the commodity space is the Banach space of bounded sequences, $l_{\infty}$, Hervés-Beloso et al. (2000) ${ }^{1}$ proved the following infinite dimensional version of Grodal's (1972) classical theorem on the veto power of small coalitions in atomless economies: If a coalition can improve upon an allocation $f$, there exists a finite number $n(f)$ such that $f$ can be blocked by a union of $n(f)$ coalitions that can be chosen to be arbitrarily small in measure and diameter. ${ }^{2}$ Here, the diameter of a coalition can be interpreted as a measure of how similar the agents in that coalition are, where similarity of agents may refer to the similarity of their predetermined characteristics such as initial endowments and/or preferences. There are three important conclusions that follow from this result. (a) An extension of Schmeidler's (1972) classical, finite dimensional result to the case of countably many commodities: Any allocation outside the core can be blocked by a coalition of an arbitrarily small measure. ${ }^{3}$ Hence, to implement a core allocation, the formation of only small coalitions is sufficient. (b) In fact, we can further restrict our attention to those small coalitions that can be represented as a union of finitely many coalitions each consisting of similar agents. Therefore, to implement a core allocation all we need to assume is the possibility of communication/coordination between the members of any finite collection of approximately homogenous coalitions, i.e., "types." (c) Given an allocation $f$ that is outside the core, we can find an upper bound, $n(f)$, to the number of types needed to block $f$, independent of the level of homogeneity and size of these types. Notice, however, that in contrast to Grodal's original result, where the number of commodities is identified as a uniform upper bound, this upper bound $n(f)$ depends on $f$, i.e., the particular allocation that must be blocked. Hence, despite the conclusion (c), to make sure that all allocations outside the core will be blocked, we may need to assume the possibility of communication between an arbitrarily large (but finite) number of types. Nevertheless, it should be noted that when the space of agents is totally bounded, for predetermined and acceptably small levels of measure and diameter, as an immediate implication of the conclusion (a), we can find an upper bound to the number of types that we need, independent of the particular allocation that must be blocked.

One of our main purposes in the present paper is to prove the following version of Grodal's (1972) theorem: If the commodity space is an ordered Banach space which has an interior point in its positive cone, provided that the space of agents is atomless and endowed with a separable pseudometric, given any positive number $\varepsilon$, an allocation outside the core can be blocked by a coalition of measure less than $\varepsilon$ that can be represented as a union of finitely many coalitions each having a diameter less than $\varepsilon$. This result immediately extends the conclusions (a) and (b) to the case of an economy with an arbitrary number of commodities so that, say, a model with continuous time or an Arrow-Debreu economy with state contingent commodities and a continuum of states can also be covered. ${ }^{4}$ On the other hand, in our extension we sacrifice the conclusion (c) which, in our opinion, does not seem to be very important. ${ }^{5}$

The method of proof that we use in this paper is substantially different than that of Hervés-Beloso et al. (2000). They work with Mackey continuous preferences so that gains/losses in the distant future are negligible. Since in their model a commodity bundle is a sequence, this allows them to disregard the tails of a blocking allocation and use Grodal's (1972) finite dimensional approach. Obviously, it is hard to imagine a similar argument that could be used in our more general model. Instead of following this approach, here we first give an extension of Schmeidler's (1972) result using Uhl's (1969) theorem on the approximate convexity of the range of an infinite dimensional, atomless vector measure. Our proof is almost the same with that of Schmeidler: The only difference is that we benefit from our lemma to be able

\footnotetext{
${ }^{1}$ We owe this reference together with Cornwall (1972) and Hervés-Beloso et al. (2005) to referees.

${ }^{2}$ Hervés-Beloso et al. (2000) define an allocation as a Gelfand integrable function, but their arguments would also work with Bochner integrable allocations.

${ }^{3}$ In fact, Schmeidler proved the following stronger result: If a coalition $E$ can improve upon an allocation $f$ via $g$, then for any positive number $c$ less than the measure of $E$, there is a subcoalition $F$ of measure $c$ that blocks the allocation $f$ via $g$. Example 1 of Hervés-Beloso et al. (2000) shows that when there are infinitely many commodities, in order to block $f$, subcoalitions may need to use alternative allocations that are possibly different than $g$. The technical question whether $c$ can be chosen to be arbitrary is not addressed in Hervés-Beloso et al. (2000). Our analysis below shows that the answer is positive.

4 This follows from the fact that the Banach space of bounded, continuous real functions on a topological space and the Banach space of essentially bounded real functions on a measure space have interior points in their positive cone under their natural ordering.

${ }^{5}$ Example 2 of Hervés-Beloso et al. (2000) shows that under our assumptions the conclusion (c) cannot be preserved. For more on this, see footnote 17 below.
} 
to use an approximate version of Schmeidler's original argument which relies on the precise convexity of the range of a finite dimensional, atomless vector measure. We then employ our lemma once again to derive our version of Grodal's theorem from the extended version of Schmeidler's theorem.

A related, classical result on finite dimensional, atomless economies is due to Vind (1972) which reads as follows: Under a suitable local non-satiation condition, an (attainable) allocation outside the core can be blocked by a coalition of an arbitrary measure. This result implies that given an allocation outside the core, we can find an arbitrarily large majority of agents who would be better off by suitably redistributing their resources among themselves, and hence, vindicates the core as a solution concept from a normative perspective. Recently, Hervés-Beloso et al. (2005, Theorem 3.3.) proved an infinite dimensional version of Vind's theorem for a differential information economy with the commodity space $l_{\infty} .{ }^{6}$ In this result, they assume that the set of agents can be partitioned into finitely many different subsets such that agents in each of these subsets are identical. More importantly, they also assume that the allocation which must be blocked has the equal treatment property. These assumptions enable them to reduce the problem at hand to a finite dimensional one, so that Liapounoff's (1940) theorem on the convexity of the range of a finite dimensional, atomless vector measure can be applied. As a side payoff of the extended version of Schmeidler's (1972) theorem, we generalize in the present paper the result of Hervés-Beloso et al. (2005) in several dimensions: (i) We cover the case of an arbitrary allocation which is outside the core. (ii) We drop the assumption that the set of agents is partitional. (iii) Instead of working on $l_{\infty}$, we assume that the commodity space is an ordered Banach space which has an interior point in its positive cone, so that models with a continuum of commodities can also be covered. (iv) We drop the assumption that preferences are convex. (v) We drop the assumption that there is a common prior. ${ }^{7}$ The economic importance of the point (i) deserves a special emphasis: We can now conclude that even with infinitely many commodities, given any allocation outside the core, an arbitrarily large majority of agents can improve upon this allocation.

It is clear that whenever core-Walras equivalence holds, the above results on the veto power of small or big coalitions can also be interpreted as arguments supporting the notion of a Walrasian equilibrium. We next turn to the issue of core-Walras equivalence. Podczeck (2003, p. 701) writes:

"Suppose $f$ is a feasible allocation of some (atomless) economy, and suppose there is a price system $p$ such that relative to every fixed separable subspace $G$ of the commodity space almost all agents are optimizing at $p$. Then, since allocations have to be almost separably valued, $f$ is a core allocation."

He then adds in footnote 9:

"It may be shown that, conversely, for a given core allocation a price system such as above exists (even when the commodity space is non-separable) provided the economy in question is atomless and, say, the "desirable assumptions" hold."

From the context we infer that these "desirable assumptions" include monotonicity of preferences and the assumption that consumption sets are equal to the positive cone which has a non-empty interior. A useful implication of Podczeck's second observation is that in the coalitional economy, which is implicit in the individualistic model, core-Walras equivalence must hold. Following Vind's (1964) coalitional approach, Cheng (1991) demonstrates this fact under the assumption that consumption sets are equal to the whole space. We introduce here a coalitionwise local nonsatiation condition (see the assumption (LNNC) below), and use this condition to give a direct and simple proof of core-Walras equivalence for the coalitional economy, without making restrictive assumptions on the consumption set correspondence. This result transforms the problem of core-Walras equivalence in the individualistic model to the problem of equivalence between coalitional equilibria and individualistic Walrasian equilibria. Thus, we arrive at an alternative interpretation of the negative examples of Tourky and Yannelis (2001) and Podczeck (2003) on core-Walras equivalence (for the individualistic model) in the case of a non-separable commodity space: At a given price vector, an allocation can be optimal for every subcoalition of a coalition, even though that allocation is suboptimal for every

\footnotetext{
${ }^{6}$ For an extension of Vind's theorem in another direction, see Sun and Yannelis (2007, Proposition 5), where the authors consider an asymmetric information economy with informationally negligible agents and finitely many contingent commodities.

${ }^{7}$ In the main body of the paper we do not model information explicitly. In Appendix A we construct a differential information economy and show that this model is compatible with our main results.
} 
agent in that coalition, since it may not be possible to aggregate the alternatives that are better at the individual level to better coalitional alternatives via the Bochner integral.

We next apply the coalitional equivalence result to give a proof of Podczeck's (2003) assertion on the existence of a price system at which on every separable subspace almost all agents are optimizing. This allows us to arrive at a second characterization of the core. Our coalitionwise local non-satiation condition is satisfied under the assumptions of Podczeck (2003, Theorem 4), and in any model where preferences are monotone and consumption sets are equal to the positive cone. Hence, our formulation of Podczeck's (2003) assertion extends, in a technical sense, core-Walras equivalence results of Rustichini and Yannelis (1991, Theorem 4.1) and Podczeck (2003, Theorem 4), for in both of these results the commodity space is assumed to be separable. Moreover, excluding convexity, we do not impose any restriction on the shape of consumption sets so that they are allowed to be "thin" and/or unbounded subsets of the positive cone. Hence, unlike the mentioned previous equivalence results, ${ }^{8}$ we can cover various models of differential information, for instance the one that we would obtain by replacing the commodity space of Hervés-Beloso et al. (2005) with an ordered, separable Banach space which has an interior point in its positive cone. Equivalence between the core (in the sense of Yannelis, 1991b) and the set of Walrasian equilibria (in the sense of Radner, 1968) for differential information economies was previously proved by Einy et al. (2001, Theorem B) for the case of finitely many commodities, and by Hervés-Beloso et al. (2005, Theorem 3.2) for the case of an economy with the commodity space $l_{\infty}$ and Mackey continuous preferences. ${ }^{9}$

Using the coalitional equivalence result, we then show that in an atomless economy the strong core coincides with the core and every core allocation is stable in the sense of Cornwall (1969): Once a core allocation takes effect, no coalition has an incentive to block that allocation.

We finally present an extension of Kannai's (1970) theorem on the continuity of the core correspondence to the case of an economy with an ordered Banach commodity space which has an interior point in its positive cone. The finite dimensional version of Kannai's theorem was previously generalized by Grodal (1971) to atomic (mixed) economies and by Hüsseinov (2003) to economies with possibly non-convex preferences. In our extension to infinitely many dimensions, we do not require convexity or non-atomicity.

The paper is organized as follows: In Section 2 we introduce our notation and terminology. The results are presented in Section 3. In the main body of the paper we do not model information explicitly and show in Appendix A that our main results are compatible with the case of a differential information economy with a finite state space and an ordered Banach commodity space which has an interior point in its positive cone.

\section{Notation and terminology}

Recall that a partial order (an antisymmetric, reflexive, transitive binary relation) $\geq$ on a vector space $\mathcal{X}$ is said to be a vector ordering if for any $x, y, z \in \mathcal{X}$ and any positive number $\alpha, x \geq y$ implies $\alpha x+z \geq \alpha y+z$. Throughout the paper, $S$ denotes an ordered Banach space, i.e., a Banach space endowed with a vector ordering $\geq$ such that the positive cone $S_{+}:=\{x \in S: x \geq 0\}$ is closed. $S^{\prime}$ stands for the norm dual of $S$. The value of a $p \in S^{\prime}$ at $x \in S$ is denoted by $\langle p, x\rangle$ instead of $p(x)$. $S_{+}^{\prime}$ stands for the positive cone of $S^{\prime}$, i.e., $S_{+}^{\prime}:=\left\{p \in S^{\prime}:\langle p, x\rangle \geq 0, \forall x \in S_{+}\right\}$.

We preserve the letters $\alpha, \gamma, \delta, \varepsilon$ for real numbers and the letters $i, j, k, m, n$ for natural numbers. $\mathbb{N}$ (resp. $\mathbb{Q}$ ) denotes the set of all natural (resp. rational) numbers.

Throughout the paper, $(T, \Sigma, \mu)$ stands for a measure space which consists of a non-empty set $T$, a $\sigma$-algebra $\Sigma$ of subsets of $T$, and a countably additive measure $\mu$ on $\Sigma$. We refer to elements of $\Sigma$ as measurable sets. $\Sigma_{E}$ denotes the restriction of $\Sigma$ to subsets of a measurable set $E$. Given a measurable set $E$, when we say that a function $f$ from $E$ into $S$ is measurable (resp. integrable) we mean that $f$ is (strongly) $\mu$-measurable (resp. Bochner $\mu$-integrable). A detailed exposition of these notions can be found in Dunford and Schwartz (1967, Chapter 3).

Let $E$ be a measurable set and take a correspondence $\Psi$ from $E$ into $S$. Graph of $\Psi$ is the set $\operatorname{Gr}_{\Psi}:=\{(t, x) \in E \times S$ : $x \in \Psi(t)\} . \int_{E} \Psi \mathrm{d} \mu$ denotes the integral of $\Psi$ over $E$, which is defined as the set of all points $x$ of the form $x=\int_{E} f \mathrm{~d} \mu$, for some integrable $f: E \rightarrow S$ with $f(t) \in \Psi(t) \mu$-almost everywhere on $E$.

\footnotetext{
${ }^{8}$ Rustichini and Yannelis (1991, Theorem 4.1) assume that consumption sets are equal to the positive cone, while Podczeck (2003, Theorem 4) assumes that consumption sets are integrably bounded.

${ }^{9}$ Sun and Yannelis (2007, Proposition 3) also prove a core-Walras equivalence result for an asymmetric information economy with informationally negligible agents and finitely many contingent commodities.
} 
The terms "almost every" and "almost everywhere" are abbreviated as "a.e.". We sometimes omit the letter $\mu$ and write (respectively) $\int_{E} f$ and a.e., instead of $\int_{E} f \mathrm{~d} \mu$ and $\mu$-a.e., and similarly for other related terms and notations.

Let $E$ and $F$ be measurable sets. $E \backslash F$ (resp. $E \Delta F$ ) denotes the set theoretic difference of $E$ from $F$ (resp. the symmetric difference of $E$ and $F)$. When $\mu(E \Delta F)=0$, we say that $E$ and $F$ are equivalent and write $E \sim F$.

Given a subset $\Theta$ of the Cartesian product of two sets $O$ and $P, \operatorname{proj}_{O} \Theta$ denotes the set $\{o \in O$ : $\exists p \in P$ such that $(o, p) \in \Theta\}$.

Let $\mathcal{X}$ be a topological space. The Borel $\sigma$-algebra of $\mathcal{X}$ is denoted by $\mathfrak{B}(\mathcal{X})$. For a subcollection $\Sigma_{0}$ of $\Sigma, \Sigma_{0} \otimes \mathfrak{B}(\mathcal{X})$ stands for the $\sigma$-algebra generated by the collection $\Sigma_{0} \times \mathfrak{B}(\mathcal{X}):=\left\{E \times Y: E \in \Sigma_{0}, Y \in \mathfrak{B}(\mathcal{X})\right\}$. Assume now $\mathcal{X}$ is endowed with a pseudometric $d$. The diameter of a set $Y \subset \mathcal{X}$ is the extended real number $\operatorname{diam} Y:=\sup \{d(x, y)$ : $x, y \in Y\}$. For any $x \in \mathcal{X}$ and any $\varepsilon>0$, the set $\{y \in \mathcal{X}: d(x, y)<\varepsilon\}$ is denoted by $B_{\varepsilon}(x)$. Given a non-empty set $Y$ and a point $x$ in $\mathcal{X}$, we define $\operatorname{dist}(x, Y):=\inf _{y \in Y} d(x, y)$. The Hausdorff distance between two non-empty sets $Y, Z$ in $\mathcal{X}$ is defined by

$$
\sigma(Y, Z):=\max \left\{\sup _{y \in Y} \operatorname{dist}(y, Z), \sup _{z \in Z} \operatorname{dist}(z, Y)\right\} .
$$

Given a subset $A$ of $S$, int $A, \operatorname{cl} A$, and $\overline{\mathrm{co}} A$ denote the interior of $A$, the closure of $A$, and the closed convex hull of $A$, respectively. Unless stated otherwise, all topological notions regarding sets and sequences in $S$ refer to the norm topology of $S$.

Let $\left\{x_{n}\right\}$ be a sequence in $S$. We denote by $w-\lim _{n} x_{n}$ the weak limit of $\left\{x_{n}\right\}$, and $w-L s_{n} x_{n}$ denotes the set of all weak limit points of $\left\{x_{n}\right\}$, i.e., $w-L s_{n} x_{n}$ is the set of all points $x$ such that $x=w-\lim _{j} x_{n_{j}}$ for a subsequence $\left\{x_{n_{j}}\right\}$ of $\left\{x_{n}\right\}$.

For a measurable set $E, g(E)$ stands for the range of a function $g$ from $E$ into $S$, that is, $g(E):=\{g(t): t \in E\}$. If $g(E)$ is a separable subset of $S$, we say that $g$ is separably valued on $E$. A function $f$ from $T$ into $S$ is said to be essentially separably valued if $f$ is separably valued on a measurable set $T^{\prime}$ such that $T^{\prime} \sim T$. $L_{1}(\mu, S)$ denotes the Banach space of (equivalence classes of) integrable functions from $T$ into $S$.

\section{The model and the results}

\subsection{The model}

The commodity space is an ordered Banach space $S .(T, \Sigma, \mu)$ denotes a measure space of consumers. Consumption sets of consumers are defined by a non-empty valued correspondence $X: T \Rightarrow S$, where $X(t)$ is the set of a priori possible consumption bundles of a consumer $t$. Endowments of consumers are represented by an integrable function $e: T \rightarrow S$, where $e(t)$ is the initial endowment of commodities of a consumer $t$. Preferences of consumers are defined by means of a correspondence $\succ: T \Rightarrow S \times S$ such that $\succ_{t} \subset X(t) \times X(t)$ for all $t \in T$. Here, $\succ_{t}$ represents the preference relation of a consumer $t$. An exchange economy, then, is a list $\xi:=\{(T, \Sigma, \mu), S, X, e, \succ\}$.

$\succsim_{t}:=\left\{(x, y) \in X(t) \times X(t):(y, x) \notin \succ_{t}\right\}$ is the preference or indifference relation of a consumer $t$. Instead of $(x, y) \in \succ_{t}$ (resp. $\left.(x, y) \in \succsim_{t}\right)$ we sometimes write $x \succ_{t} y$ (resp. $x \succsim_{t} y$ ). An allocation $f$ is an integrable function from $T$ into $S$ such that $f(t) \in X(t)$ a.e. on $T$. For an allocation $f, U_{f}$ denotes the correspondence from $T$ to $S$ defined by $U_{f}(t):=\left\{x \in X(t): x \succ_{t} f(t)\right\}$ for all $t \in T$.

We now present the pool of assumptions that we use throughout the paper.

(A0). $S$ is an ordered Banach space with int $S_{+} \neq \emptyset .(T, \Sigma, \mu)$ is a positive, finite and complete measure space.

(A1). $X(t)$ is convex for every $t \in T$, and $\mathrm{Gr}_{X}$ belongs to $\Sigma \otimes \mathfrak{B}(S)$.

(A2). $X(t)$ is closed for every $t \in T$.

(A3) (Survival). There is an integrable function $\varphi: T \rightarrow S$ such that $\varphi(t) \in X(t)$ a.e. on $T$, and $\int_{E}(e-\varphi) \mathrm{d} \mu \in \operatorname{int} S_{+}$ for every measurable set $E$ with $\mu(E)>0$. 
(P1) (Measurable preferences). For any allocation $f$, and any separable, closed, linear subspace $Y$ of $S$ with $f(T) \subset Y$, the graph of the correspondence $Y \cap U_{f}: t \Rightarrow Y \cap U_{f}(t)(t \in T)$ belongs to $\Sigma \otimes \mathfrak{B}(Y)$.

(P2) (Upper continuity). For any $t \in T$ and any $x \in X(t)$, the set $\left\{y \in X(t): y \succ_{t} x\right\}$ is (norm) open in $X(t)$.

(P3) (Lower continuity in the weak topology). For any $t \in T$ and any $x \in X(t)$, the set $\left\{y \in X(t): x \succ_{t} y\right\}$ is weakly open in $X(t)$.

(P4) (Ordered preferences). For any $t \in T, \succ_{t}$ is asymmetric $\left((x, y) \in \succ_{t}\right.$ implies $\left.(y, x) \notin \succ_{t}\right)$ and negatively transitive $\left((y, x) \notin \succ_{t}\right.$ and $(x, z) \notin \succ_{t}$ imply $\left.(y, z) \notin \succ_{t}\right)$. In particular, $\succsim_{t}$ is reflexive, complete and transitive.

Remark 1. The condition that the positive cone has an interior point holds in the models of Hervés-Beloso et al. (2000, 2005) (see footnote 12 below and Appendix A), and directly assumed in Khan and Yannelis (1991), Rustichini and Yannelis (1991, Theorem 4.1), and Podczeck (2003). The remaining conditions in assumption (A0) are standard. In the sequel, (A0) is assumed to be true without further mention.

Remark 2. (A1) is either trivially true or directly assumed in Khan and Yannelis (1991), Rustichini and Yannelis (1991), Hervés-Beloso et al. (2000), and Podczeck (2003). Moreover, one can map the model of Hervés-Beloso et al. (2005) into our setting and show that (A1) also holds in their model (see Appendix A).

Remark 3. (A2), (P2) and (P4) are standard assumptions.

Remark 4. The lower continuity condition (P3) will be used only in the extension of Kannai's (1970) theorem.

Remark 5. (A3) is an abstraction of the survival assumption (H.2) of Hervés-Beloso et al. (2000); in particular, in their model (A3) holds. Notice that if $X$ admits an integrable selection $\varphi$ with $e(t)-\varphi(t) \in$ int $S_{+}$for a.e. $t \in T$, then (A3) holds. ${ }^{10}$ Hence, (A3) is valid, if as in Hervés-Beloso et al. (2005), $0 \in X(t)$ and $e(t) \in \operatorname{int} S_{+}$for every $t \in T$. The following assumption employed in Khan and Yannelis (1991) and Podczeck (2003, Theorems 2 and 4) also implies (A3):

(R-5.1). $\operatorname{Gr}_{X}$ belongs to $\Sigma \otimes \mathfrak{B}(S), X$ is integrably bounded ${ }^{11}$ and there exists a separable subset $S_{0}$ of $S$ such that $\left[e(t)-S_{0} \cap X(t)\right] \cap$ int $S_{+}$is non-empty a.e. on $T$.

To see that (R-5.1) indeed implies (A3), ignoring a set of measure 0 assume $e(T)$ is separable and let $Y$ be the closed, linear space spanned by $e(T) \cup S_{0}$. Define a correspondence $\Psi: t \Rightarrow[e(t)-Y \cap X(t)] \cap$ int $S_{+}$from $T$ into $Y$. Now, note that since $\mathrm{Gr}_{X}$ is in $\Sigma \otimes \mathfrak{B}(S), \mathrm{Gr}_{\Psi}$ belongs to $\Sigma \otimes \mathfrak{B}(Y)$. Since $Y$ is separable and complete, and since $\Psi$ is non-empty valued, by Aumann's (1969) measurable selection theorem, $\Psi$ admits a measurable selection $h$. Since $X$ is integrably bounded, the mapping $\varphi:=e-h$ satisfies all conditions demanded by (A3).

Remark 6. The following condition implies (P1):

$\operatorname{Gr}_{X}$ belongs to $\Sigma \otimes \mathfrak{B}\left(S_{+}\right)$, and $\succ$ is induced by a Carathéodory function $U(\cdot, \cdot)$ on $T \times S_{+}$; that is, for every $t \in T, \succ_{t}$ is induced by a norm continuous real function $U(t, \cdot)$ on $S_{+}$, such that for every fixed $x \in S_{+}$the mapping $t \rightarrow U(t, x)$ is measurable.

To see this point, let $f$ be an allocation and $Y$ be a separable, closed subspace of $S$ with $f(T) \subset Y$. Since $U(\cdot, \cdot)$ is a Carathéodory function, using the fact that $f$ is the pointwise limit of a sequence of simple functions, it can easily be seen that the mapping $t \rightarrow U(t, f(t))(t \in T)$ is measurable. Thus, the function $\phi:(t, x) \rightarrow U(t, x)-U(t, f(t))$ is a Carathéodory function on $T \times S_{+}$, and so is the restriction $\phi_{0}$ of $\phi$ to $T \times\left(S_{+} \cap Y\right)$. Since $Y$ is separable, $\phi_{0}$ must be jointly measurable, i.e., $\Sigma \otimes \mathfrak{B}\left(S_{+} \cap Y\right)$-measurable (see Aliprantis and Border, 1999, Lemma 4.50, p. 151).

\footnotetext{
10 The discussion that follows (H.2) in Hervés-Beloso et al. (2000) shows that the converse is not true, that is, (A3) does not imply the condition $e(t)-\varphi(t) \in \operatorname{int} S_{+}$for a.e. $t \in T$.

11 That is, there exists an integrable function $q: T \rightarrow \mathbb{R}$ such that $\sup \{\|x\|: x \in X(t)\} \leq q(t)$ a.e. on $T$.
} 
Thus, in this case, $\operatorname{Gr}_{Y \cap U_{f}}=\phi_{0}^{-1}(0, \infty) \cap \mathrm{Gr}_{X}$ belongs to $\Sigma \otimes \mathfrak{B}\left(S_{+} \cap Y\right)$, which proves our claim. Also note that $\operatorname{Gr}_{Y \cap U_{f}}=(T \times Y) \cap \mathrm{Gr}_{U_{f}}$. Hence, (P1) is again valid, if for any allocation $g$ the set $\operatorname{Gr}_{U_{g}}$ belongs to $\Sigma \otimes \mathfrak{B}(S)$. Following Podczeck (2003, Appendix A), we also note that if $X$ is graph measurable and integrably bounded, under some further mild assumptions, the following condition (Aumann measurability) implies (P1):

For any two allocations $g$ and $h$, the set $\left\{t \in T: g(t) \succ_{t} h(t)\right\}$ belongs to $\Sigma$.

In particular, (P1) is valid in Podczeck (2003, Theorems 2 and 4). Khan and Yannelis (1991) assume $\mathrm{Gr}_{\succ} \in \Sigma \otimes \mathfrak{B}(S \times$ $S$ ), which obviously implies Aumann measurability. So, by the above observation, (P1) is also valid in their model. It can also be shown that the models of Hervés-Beloso et al. $(2000,2005)$ satisfy (P1) as well. ${ }^{12}$ (See also Appendix A.) Finally, note that for any two allocations $g$ and $h$, we have $\left\{t \in T: g(t) \succ_{t} h(t)\right\}=\operatorname{proj}_{T}\left(\mathrm{Gr}_{g} \cap \mathrm{Gr}_{Y^{\prime} \cap U_{h}}\right)$, where, ignoring a null set, we assume that $Y^{\prime}$ is a separable, closed, linear subspace with $g(T) \cup h(T) \subset Y^{\prime}$. Hence, $(\mathrm{P} 1)$ is stronger than Aumann measurability.

Following Khan and Yannelis (1991), Hervés-Beloso et al. (2000, 2005), and Podczeck (2003), we assume free disposal. Hence, an allocation $f$ is attainable if $\int_{T} f \mathrm{~d} \mu \leq \int_{T} e \mathrm{~d} \mu$. A coalition $E$ is an element of $\Sigma$ with $\mu(E)>0$. $E_{0}$ is a subcoalition of a coalition $E$ if $E_{0}$ is itself a coalition and $E_{0} \subset E$. A coalition $E$ is said to block an allocation $f$ via $g$ if there exists an integrable function $g: E \rightarrow S$ such that $\int_{E} g \mathrm{~d} \mu \leq \int_{E} e \mathrm{~d} \mu$ and $g(t) \succ_{t} f(t)$ a.e. on $E$. An allocation is a core allocation if it is attainable and if it is not blocked by any coalition. The core, denoted by $\mathcal{C}(\xi)$, is the set of all core allocations. An attainable allocation $f$ belongs to the strong core, denoted by $\mathcal{S C}(\xi)$, if and only if there do not exist a coalition $E$ and an integrable function $g: E \rightarrow S$ with $\int_{E} g \mathrm{~d} \mu \leq \int_{E} e \mathrm{~d} \mu$ such that $g(t) \succsim_{t} f(t)$ a.e. on $E$ and $g(t) \succ_{t} f(t)$ a.e. on some subcoalition $E_{0}$ of $E$.

Remark 7. If preferences are ordered and monotone in the sense that, for all $t \in T, X(t)+S_{+} \subset X(t)$ and $x \succ_{t} y$ whenever $x-y \in S_{+} \backslash\{0\}$ and $y \in X(t)$, then allowing free disposal is innocuous: In the above definitions, we could replace the inequality sign " $\leq$ " with " =" and all of our results would remain true.

\subsection{A technical observation}

In this section, we discuss and prove the following lemma which shows that if a coalition blocks an allocation, it can do this, in fact, by disposing a strictly positive amount of its resources. In the remainder of the paper, this observation and an implication of its proof will be our main tolls.

Lemma 1. Let $\xi$ be an economy that satisfies assumptions (A0), (A1), (A3), (P1) and (P2). If a coalition E blocks an allocation $f$, then $\int_{E} e \mathrm{~d} \mu-z \in \int_{E} U_{f} \mathrm{~d} \mu$ for some $z \in \operatorname{int} S_{+}$.

A close relative of Lemma 1 is Theorem 9 of Cornwall (1972) which, adapted to our economic setting, reads as follows:

Suppose that $S$ is separable and $(T, \Sigma, \mu)$ is $\sigma$-finite. Let $\xi$ be an economy that satisfies assumptions (A1), (P1) and (P2). Let e $(t)$ be in int $X(t)$ a.e. on T. Take any allocation $f$ and assume that $U_{f}$ is convex valued. Now, if a coalition $E$ blocks $f$ via a function $g: E \rightarrow S$ with $\int_{E} g \mathrm{~d} \mu=\int_{E} e \mathrm{~d} \mu$, then $\int_{E} e \mathrm{~d} \mu \in \operatorname{int} \int_{E} U_{f} \mathrm{~d} \mu$.

A finite dimensional version of Cornwall's theorem was previously proved by Grodal (1971). We find another finite dimensional version of this theorem in Cornwall (1970) within the context of set valued measures. ${ }^{13}$ Compared with Cornwall's (1972) result, Lemma 1 has three advantages: (a) It does not require $S$ to be separable. (b) $U_{f}$ need not be convex valued, that is, preferences need not be convex. (c) The interior of $X(t)$ can be empty for any $t \in T$. The importance of the point (c) is based on two reasons. First, as we shall see in Appendix A, in differential information economies

\footnotetext{
12 In Hervés-Beloso et al. (2000), $S=l_{\infty}$ and $X(t)=S_{+}$for every $t \in T$. They assume preferences are induced by a function $U(\cdot, \cdot)$ on $T \times S_{+}$ with $U(t, \cdot) \in \mathcal{C}$ for every $t$, such that $t \rightarrow U(t, \cdot)$ is a measurable function from $T$ into $\mathcal{C}$, where $\mathcal{C}$ is the space of Mackey $\tau\left(l_{\infty}, l_{1}\right)$ continuous real functions on $S_{+}$which is endowed with the topology of uniform convergence on bounded subsets of $S_{+}$. Note that for every $x \in S_{+}$and every real $\alpha,\{t \in T: U(t, x)>\alpha\}=\left\{t \in T: U(t, \cdot) \in O_{x, \alpha}\right\}$, where $O_{x, \alpha}:=\{V \in \mathcal{C}: V(x)>\alpha\}$, which is open in $\mathcal{C}$. Hence, by measurability of $t \rightarrow U(t, \cdot)$, the function $t \rightarrow U(t, x)$ is measurable for every $x$. Finally, since $\tau\left(l_{\infty}, l_{1}\right)$ is weaker than the norm topology, it follows that $U(\cdot, \cdot)$ is a Carathéodory function. Thus, (P1) is valid in this framework.

13 The main contribution of the result presented in Cornwall (1970) is its role in the proof of Cornwall's (1969) extension of the coalitional equivalence theorem of Vind (1964), which inspired the equivalence result that we prove in this paper.
} 
consumption choices of agents must be compatible with the information available to them. This informational constraint typically leads to "thin" consumption sets which have an empty interior even if the positive cone has an interior point. Second, in equilibrium-core existence results, it is frequently assumed that consumption sets are weakly compact (e.g., see Khan and Yannelis (1991), Martins-da-Rocha (2003), or Podczeck (2003)). On the other hand, a Banach space admits weakly compact sets with interior points if and only if the space under focus is reflexive. ${ }^{14,15}$

We proceed with a proof of Lemma 1.

Proof of Lemma 1. Let $g: E \rightarrow S$ be an integrable function with $\int_{E}(g-e) \leq 0$ such that $g(t) \in U_{f}(t)$ a.e. on $E$. Ignoring a null set, assume that there is a separable, closed, linear subspace $Y$ which contains the set $f(E) \cup g(E) \cup$ $\varphi(E)$, where the function $\varphi$ is as in the survival assumption (A3). For each $\varepsilon>0$, define a correspondence $\hat{B}_{\varepsilon}$ by $\hat{B}_{\varepsilon}(t):=Y \cap B_{\varepsilon}(g(t))(t \in E)$ and note that $\operatorname{Gr}_{\hat{B}_{\varepsilon}}$ belongs to $\Sigma_{E} \otimes \mathfrak{B}(Y)$. By measurability assumptions (A1) and (P1), clearly, graphs of the correspondences $\hat{X}(t):=Y \cap X(t), \hat{U}_{f}(t):=Y \cap U_{f}(t)(t \in E)$ also belong to $\Sigma_{E} \otimes \mathfrak{B}(Y)$.

For every $t \in E$, put $\varepsilon_{t}:=\sup \left\{\varepsilon>0:\left(\hat{B}_{\varepsilon}(t) \cap \hat{X}(t)\right) \subset \hat{U}_{f}(t)\right\}$. Obviously, by the continuity assumption (P2), $\varepsilon_{t}>0$ a.e. on $E$. Now note that for any $\alpha>0$, we have

$$
\left\{t \in E: \varepsilon_{t}<\alpha\right\}=\bigcup_{r \in \mathbb{Q} \cap(0, \alpha)}\left\{t \in E: \hat{B}_{r}(t) \cap \hat{X}(t) \cap\left(Y \backslash \hat{U}_{f}(t)\right) \neq \emptyset\right\}=\operatorname{proj}_{E} \Theta,
$$

where $\Theta:=\left(\bigcup_{r \in \mathbb{Q} \cap(0, \alpha)} \mathrm{Gr}_{\hat{B}_{r}}\right) \cap \operatorname{Gr}_{\hat{X}} \cap\left(E \times Y \backslash \mathrm{Gr}_{\hat{U}_{f}}\right)$, which obviously belongs to $\Sigma_{E} \otimes \mathfrak{B}(Y)$. Now, since $Y$ is separable and complete, from the projection theorem (see Hu and Papageorgiou, 1997, Theorem 1.33, p. 149) it follows that the mapping $t \rightarrow \varepsilon_{t}$ is measurable.

For each $n \in \mathbb{N}$, put $h_{n}:=g+(1 / n)(\varphi-g)$, and $E_{n}:=\left\{t \in E:\left\|h_{n}(t)-g(t)\right\|<\varepsilon_{t}\right\}$. Note that $E_{n} \in \Sigma, E_{n} \subset E_{n+1}$ $(n \in \mathbb{N})$ and $\bigcup_{n} E_{n} \sim E$. Hence, $\lim _{n} \mu\left(E \backslash E_{n}\right)=0$. For each $n \in \mathbb{N}$, define the function $g_{n}: E \rightarrow Y$ by

$$
g_{n}(t):= \begin{cases}g(t) & \text { for } t \in E \backslash E_{n}, \\ h_{n}(t) & \text { for } t \in E_{n} .\end{cases}
$$

Since $X$ is convex valued, by construction, $g_{n}(t) \succ_{t} f(t)$ a.e. on $E$. Now note that

$$
\begin{aligned}
\int_{E} g_{n} & =\int_{E \backslash E_{n}} g+\int_{E_{n}} h_{n}=\int_{E \backslash E_{n}}\left(g-h_{n}\right)+\int_{E} h_{n} \\
& =\int_{E \backslash E_{n}} \frac{1}{n}(g-\varphi)+\int_{E}\left(\left(1-\frac{1}{n}\right) g+\frac{1}{n} \varphi\right) \\
& \leq \int_{E \backslash E_{n}} \frac{1}{n}(g-\varphi)+\int_{E}\left(\left(1-\frac{1}{n}\right) e+\frac{1}{n} \varphi\right) \\
& =\int_{E} e+\frac{1}{n} u_{n},
\end{aligned}
$$

where $u_{n}:=\int_{E}(\varphi-e)+\int_{E \backslash E_{n}}(g-\varphi)$. Since $\int_{E}(\varphi-e) \in-$ int $S_{+}$, from absolute continuity of integral it follows that $u_{n}$ is in - int $S_{+}$for a sufficiently large $n$. Hence, $z:=\int_{E} e-\int_{E} g_{n}$ belongs to int $S_{+}$.

The following result is implicitly proved above. This will play a key role in the proof of coalitional core-Walras equivalence.

Corollary 1. Let $\xi$ be an economy that satisfies assumptions (A0), (A1), (A3), (P1) and (P2). Let f be an allocation, and $g: E \rightarrow S$ be an integrable function such that $g(t) \succ_{t} f(t)$ a.e. on a coalition $E$. Then, there exist a number $\alpha>0$

\footnotetext{
14 See Dunford and Schwartz (1967, Theorem V.4.7).

15 We are not aware of an infinite dimensional reflexive Banach space which is used in applied economic theory and which has an interior point in its positive cone under its natural ordering. Hence, Cornwall's (1972) theorem seems to be practically incompatible with the assumption that consumption sets are weakly compact subsets of the positive cone. On the other hand, this interiority problem also leaves many important cases out of the coverage of Lemma 1: Not only many important reflexive spaces such as $L_{p}$ spaces $(1<p<\infty)$, i.e., spaces of $p$-power integrable functions, but also $L_{1}$ spaces, as well as spaces of signed measures cannot be covered under their natural ordering.
} 
and a subcoalition $F$ of $E$ such that the point $\int_{E} g \mathrm{~d} \mu+\alpha \int_{F}(\varphi-g) \mathrm{d} \mu$ belongs to the set $\int_{E} U_{f} \mathrm{~d} \mu$, where $\varphi$ is as in assumption (A3).

\subsection{Decisive power of small or big coalitions}

In this part of the paper we show that, without changing the core, there are various ways in which we can restrict the set of coalitions that are allowed to form. We now introduce a condition needed for the extension of Vind's (1972) theorem.

Definition 1. We say that an allocation $f$ is coalitionwise locally non-satiating if $\int_{E} f \mathrm{~d} \mu \in \operatorname{cl} \int_{E} U_{f} \mathrm{~d} \mu$ for every coalition $E$.

Remark 8. Obviously, if preferences are monotone (see Remark 7), any allocation is coalitionwise locally nonsatiating. In fact, the following weaker condition, which requires the existence of a feasible improving direction, is clearly sufficient for this purpose:

(R-8.1). There is a $z \in S_{+}$such that for every $t \in T$, every $x \in X(t)$, and every $\gamma>0, x+\gamma z \in X(t)$ and $x+\gamma z \succ_{t} x$.

We next give a further case where a given allocation $f$ would be coalitionwise locally non-satiating:

Suppose that $\bigcup_{t \in T} X(t)$ is separable and that for every $t \in T$ preferences are locally non-satiated at $f(t) .{ }^{16}$ Then, under the measurability assumption (P1), the correspondence $t \Rightarrow B_{\varepsilon}(f(t)) \cap U_{f}(t)(t \in T)$ admits a measurable selection for every $\varepsilon>0$. This selection is integrable, for $\mu$ is finite and $f$ is integrable.

We are now ready to present the promised extensions of Schmeidler's (1972) and Vind's (1972) theorems.

Theorem 1. Let $\xi$ be an economy that satisfies assumptions (A0), (A1), (A3), (P1) and (P2). Suppose that a coalition E blocks an allocation $f$. If $\mu$ is atomless, the following are true.

(a) For any $c \in(0, \mu(E))$, there is a subcoalition $E_{0}$ of $E$ with $\mu\left(E_{0}\right)=c$ that blocks $f$.

(b) If $f$ is attainable and coalitionwise locally non-satiating, then, for any $c \in(0, \mu(T))$, there is a coalition $F$ with $\mu(F)=c$ that blocks $f$.

Remark 9. In Appendix A, we note that the condition (R-8.1) is valid in Hervés-Beloso et al. (2005). Hence, in their model every allocation is coalitionwise locally non-satiating. Thus, their Theorem 3.3 is a particular case of Theorem $1(b)$.

Proof of Theorem 1. By Lemma 1, there exists an integrable function $g: E \rightarrow S$ such that $g(t) \succ_{t} f(t)$ a.e. on $E$ and $z:=\int_{E}(e-g) \in$ int $S_{+}$. First, pick any $c \in(0, \mu(E))$. Put $C:=\operatorname{cl}\left\{\left(\mu(B), \int_{B} e-g\right): B \subset E, B \in \Sigma\right\}$. By Uhl's (1969) theorem (see also his concluding remark), $C$ is convex. Hence, there exists a sequence of measurable subsets $\left\{B_{n}\right\}$ of $E$ such that $\lim _{n}\left(\mu\left(B_{n}\right), \int_{B_{n}} e-g\right)=\gamma(\mu(E), z)$, where $\gamma:=c / \mu(E)$. Since $\mu$ is atomless, for each $n$, there exists a measurable subset $E_{n}$ of $E$ such that $\mu\left(E_{n}\right)=c$ and $\mu\left(E_{n} \Delta B_{n}\right)=\left|c-\mu\left(B_{n}\right)\right|$. Since $\lim _{n} \mu\left(E_{n} \Delta B_{n}\right)=0$, by absolute continuity of integral, $\lim _{n} \int_{E_{n}}(e-g)=\gamma z$. Since $\gamma z \in$ int $S_{+}$, for a sufficiently large $n, \int_{E_{n}}(e-g)$ belongs to int $S_{+}$. Hence, the coalition $E_{n}$ blocks $f$ via $g$. This proves part (a).

Now assume $f$ is attainable and coalitionwise locally non-satiating. By part (a), to complete the proof it suffices to show that there exists a blocking coalition of arbitrarily large measure. Let $U \subset S$ be an open set with $0 \in U$ such that $\varepsilon z-U \subset$ int $S_{+}$, where $\varepsilon$ is a number in $(0,1)$. Following Yannelis (1991a, Theorem 6.2), cl $\int_{E} U_{f}$ is convex. Since, by assumption, $\int_{E} f$ belongs to $\mathrm{cl} \int_{E} U_{f}$, there exists an integrable function $h: E \rightarrow S$ such that $h(t) \succ_{t} f(t)$ a.e. on $E$ and $\int_{E} h=\varepsilon \int_{E} g+(1-\varepsilon) \int_{E} f+u$ for some $u \in U$. Then, $\int_{E} h=\varepsilon \int_{E} e+(1-\varepsilon) \int_{E} f-(\varepsilon z-u)$. Note that $z_{0}:=\varepsilon z-u$ belongs to int $S_{+}$. Let $V_{1}, V_{2}, V_{3} \subset S$ be open sets with $0 \in V_{1} \cap V_{2} \cap V_{3}$ such that $z_{0}-V_{1}-V_{2}-V_{3} \subset$

\footnotetext{
${ }^{16}$ Recall that $x \in X(t)$ is said to be a satiation point if the set $\left\{y \in X(t): y \succ_{t} x\right\}$ is empty. The preference $\succ_{t}$ is said to be locally non-satiated at $x \in X(t)$ if for any neighborhood $U$ of $x$ there is a point $y \in U \cap X(t)$ such that $y \succ_{t} x$.
} 
int $S_{+}$. Since cl $\left\{\left(\mu(B), \int_{B} f, \int_{B} e\right): B \subset T \backslash E, B \in \Sigma\right\}$ is convex, there exists a measurable set $B \subset T \backslash E$ such that $v_{1}:=\int_{B} f-(1-\varepsilon) \int_{T \backslash E} f \in V_{1}$ and $v_{2}:=(1-\varepsilon) \int_{T \backslash E} e-\int_{B} e \in V_{2}$. As in the proof of part (a), without loss of generality we can assume $\mu(B)=(1-\varepsilon) \mu(T \backslash E)$. Furthermore, there exists an integrable function $f_{0}: B \rightarrow S$ such that $f_{0}(t) \succ_{t} f(t)$ a.e. on $B$ and $v_{3}:=\int_{B} f_{0}-\int_{B} f \in V_{3}$. Now define $l: E \cup B \rightarrow S$ by

$$
l(t):= \begin{cases}h(t) & \text { for } t \in E, \\ f_{0}(t) & \text { for } t \in B .\end{cases}
$$

Then,

$$
\begin{aligned}
\int_{E \cup B} l & =\varepsilon \int_{E} e+(1-\varepsilon) \int_{E} f-z_{0}+\int_{B} f+v_{3} \\
& =\varepsilon \int_{E} e+(1-\varepsilon) \int_{E} f-z_{0}+(1-\varepsilon) \int_{T \backslash E} f+v_{1}+v_{3} \\
& \leq \varepsilon \int_{E} e+(1-\varepsilon) \int_{T} e-\left(z_{0}-v_{1}-v_{3}\right) \\
& =\int_{E} e+\int_{B} e-\left(z_{0}-v_{1}-v_{2}-v_{3}\right) .
\end{aligned}
$$

Since $z_{0}-v_{1}-v_{2}-v_{3} \in \operatorname{int} S_{+}, E \cup B$ blocks $f$ via $l$. Finally, note that $\mu(E \cup B)=\mu(T)-\varepsilon \mu(T \backslash E)$.

The next result shows that even in atomic economies the precise formation of a particular coalition is unnecessary. A finite dimensional version of this result is due to Hüsseinov (2003).

Proposition 1. Let $\xi$ be an economy that satisfies assumptions (A0), (A1), (A3), (P1) and (P2), and suppose that a coalition $E$ blocks an allocation $f$. Then, there exist a $\delta>0$ and a function $g: E \rightarrow S$ such that any subcoalition $F$ of $E$ with $\mu(E \backslash F)<\delta$ blocks $f$ via $g$. If $f$ is coalitionwise locally non-satiating, there is a $\delta^{\prime}>0$ such that any coalition $F$ with $\mu(E \Delta F)<\delta^{\prime}$ blocks $f$.

Proof. By Lemma 1, $E$ blocks $f$ via a function $g: E \rightarrow S$ such that $z:=\int_{E}(e-g) \in \operatorname{int} S_{+}$. Let $\left\{E_{n}\right\}$ be a sequence of subcoalitions of $E$ such that $\lim _{n} \mu\left(E \backslash E_{n}\right)=0$. By absolute continuity of integral, $\lim _{n} \int_{E_{n}}(e-g)=z$. Hence, for all sufficiently large $n, \int_{E_{n}}(e-g) \in$ int $S_{+}$, and $E_{n}$ blocks $f$ via $g$. This proves the first part. To prove the second part, let $\left\{F_{n}\right\}$ be a sequence of coalitions such that $\lim _{n} \mu\left(F_{n} \Delta E\right)=0$. Without loss of generality assume $\mu\left(F_{n} \backslash E\right)>0$ for each $n$, and pick a function $f_{n}: F_{n} \backslash E \rightarrow S$ such that $f_{n}(t) \succ_{t} f(t)$ a.e. on $F_{n} \backslash E$ and $\left\|\int_{F_{n} \backslash E}\left(f_{n}-f\right)\right\|<1 / n$. Then, $\lim _{n} \int_{F_{n} \backslash E} f_{n}=\lim _{n} \int_{F_{n} \backslash E} f=0$. Hence, if we let $g_{n}:=g$ on $F_{n} \cap E$ and $g_{n}:=f_{n}$ on $F_{n} \backslash E$, we will have $\lim _{n} \int_{F_{n}}\left(g_{n}-e\right)=-z$. So, for all sufficiently large $n, F_{n}$ blocks $f$ via $g_{n}$.

Our next purpose is to present the promised extension of Grodal's (1972) theorem. Here we assume that the set of agents is endowed with a separable topology induced by a pseudometric. One way of obtaining such a pseudometric is to derive it from a separable and metrizable topology on the set of agents' characteristics $\bigcup_{t \in T}\left(e(t)\right.$, $\left.\succsim_{t}\right)$. For a discussion of alternative topologies which can be defined on a collection of subsets of a topological space, we refer to Hu and Papageorgiou (1997, Section 1.1).

Corollary 2. Let $\xi$ be an economy that satisfies assumptions (A0), (A1), (A3), (P1) and (P2), and suppose that a coalition Eblocks an allocationf. Assume that $T$ is endowed with a pseudometric which makes T a separable topological space such that $\mathfrak{B}(T) \subset \Sigma$. Assume further that $\mu$ is atomless. Then, for any $\varepsilon, \delta>0$, there exists a subcoalition $F$ of $E$ which blocks $f$ such that $\mu(F) \leq \varepsilon$ and $F=\bigcup_{i=1}^{n} F_{i}$ for a finite collection of coalitions $\left\{F_{1}, \ldots, F_{n}\right\}$ with $\operatorname{diam} F_{i} \leq \delta$ for every $i=1, \ldots, n$.

In this result, we can interpret each $F_{i}$ as a particular "type" of consumers, and $n$ as the number of different types needed to block an allocation. Corollary 2 implies, as a consequence of Theorem 1(a), that we can control the measure of the coalition formed by the union of these different types without a difficulty. However, as Grodal (1972) emphasizes, even with finitely many commodities we may not be able to control the diameter of this union, i.e., we may truly need types that are substantially different than one another. Another issue is the number of types which must come together. 
As we noted earlier, Grodal finds a uniform upper bound to the number of types needed. On the other hand, for a given allocation $f$ that is outside the core, Hervés-Beloso et al. (2000) find an upper bound to the number of types needed to block $f$, which possibly depends on $f$, but which is uniform in diameter of types. In Corollary 2, we also loose this uniformity. ${ }^{17}$ Ignoring this difference, Theorem 1 of Hervés-Beloso et al. (2000) is a particular case of Corollary 2. When passing, we emphasize once again that if $T$ is totally bounded, we can obtain an alternative, and perhaps, a more useful uniformity as an immediate implication of Theorem 1(a): For predetermined, acceptably small levels of diameter and measure, we can choose an upper bound to number of types uniformly in allocations.

Proof of Corollary 2. By Theorem 1(a), there exists a subcoalition $E_{0}$ of $E$ with $\mu\left(E_{0}\right) \leq \varepsilon$ that blocks $f$. Let $\left\{t_{i}: i \in \mathbb{N}\right\}$ be a dense subset of $T$. Put $F_{i}:=E_{0} \cap B_{\delta / 2}\left(t_{i}\right)$ for all $i \in \mathbb{N}$. Since $\bigcup_{n=1}^{\infty} \bigcup_{i \leq n} F_{i}=E_{0}$, by Proposition 1 , for a sufficiently large $n$ the coalition $F:=\bigcup_{i \leq n} F_{i}$ blocks $f$.

From the proof of Corollary 2 it is clear that even if $\mu$ is atomic, an allocation outside the core can in fact be blocked by a union of finitely many coalitions that can be chosen to be arbitrarily small in diameter. We close this section with a related result for atomic economies. This result generalizes Proposition 1 of Hervés-Beloso et al. (2000) to an atomic economy with an arbitrary number of commodities.

Corollary 3. Let $\xi$ be an economy that satisfies assumptions (A0), (A1), (A3), (P1) and (P2), and suppose that a coalition E blocks an allocation $f$. Assume that $T$ is a Polish space such that the completion of $\mathfrak{B}(T)$ with respect to $\mu$ is $\Sigma$. Then, there exists a compact, positive measure set $K \subset E$ that blocks $f$ via a function $g: K \rightarrow S$ such that both fand $g$ are continuous on $K^{18}$

Proof. By Proposition 1, there exist a $\delta>0$ and a function $g: E \rightarrow S$ such that every coalition $F \subset E$ with $\mu(E \backslash$ $F)<\delta$ blocks $f$ via $g$. By passing to an equivalent subcoalition of $E$ if necessary, assume that $f$ and $g$ are separably valued on $E$. Since a finite Borel measure on a Polish space is tight, there is a compact, positive measure subset $K_{1}$ of $E$ with $\mu\left(E \backslash K_{1}\right)<\delta$. Since $K_{1}$ is also a Polish space and since $f$ and $g$ are separably valued on $K_{1}$, by Lusin's theorem (see Aliprantis and Border, 1999, Theorem 10.8, p. 371), we can find a compact subset $K$ of $K_{1}$, arbitrarily large in measure, such that $f$ and $g$ are continuous on $K$. In particular, we can choose $K$ such that $\mu(E \backslash K)<\delta$ and $\mu(K)>0$.

\subsection{Core-Walras equivalence and stability}

Our coalitional core-Walras equivalence result is based on the following local non-satiation condition.

(LNNC) (Local non-satiation at non-satiated coalitions). For every allocation $f$ and every coalition $E$, if $\int_{E} U_{f} \mathrm{~d} \mu \neq \emptyset$, then $\int_{E} f \mathrm{~d} \mu \in \mathrm{cl} \int_{E} U_{f} \mathrm{~d} \mu$. If $\int_{E} U_{f} \mathrm{~d} \mu=\emptyset$, then there exists a subcoalition $E_{0}$ of $E$ such that $\int_{E_{0}} f \mathrm{~d} \mu \geq \int_{E_{0}} e \mathrm{~d} \mu$.

Remark 10. The assumption (LNNC) is closely related with the following well known non-satiation assumption which is also used in Podczeck's (2003) core-Walras equivalence result Theorem 4.

(R-10.1). For a.e. $t \in T$ and every $x \in X(t)$, if $x$ is not a satiation point, then $x$ is in the closure of $\left\{y \in X(t): y \succ_{t} x\right\}$. If $x$ is a satiation point, then $x \geq e(t)$.

Indeed, if as in Podczeck (2003, Theorem 4) the set $\bigcup_{t \in T} X(t)$ is separable, under the measurability assumption (P1), (R-10.1) implies that for any allocation $f$ and any coalition $E$, the set $\int_{E} U_{f} \mathrm{~d} \mu$ is empty if and only if $f(t)$ is a satiation point for a.e. $t$ in some set of positive measure $E_{0} \subset E$. Hence, in this case, (LNNC) follows from (R-10.1). ${ }^{19,20}$ On

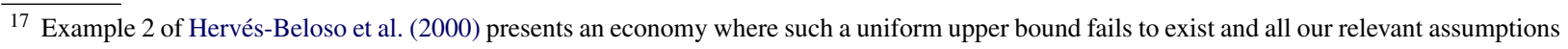
hold. They identify the source of the problem as the lack of Mackey continuity. Hence, if possible, to obtain such an upper bound in the present framework one would at least have to strengthen our continuity condition.

${ }^{18}$ If, as in Hervés-Beloso et al. (2000), each agent $t$ is endowed with a utility function $U(t, \cdot)$ such that $t \rightarrow U(t, \cdot)$ is a measurable mapping from $T$ into a second countable space $\mathcal{C}$, we can make sure that this mapping is also continuous on $K$.

19 See also Remark 8.

${ }^{20}$ We did not check whether the converse implication is also true.
} 
the other hand, in the non-separable case (R-10.1) may not imply (LNNC). ${ }^{21}$ Independent of the separability of the set $\bigcup_{t \in T} X(t)$, provided that there is a feasible improving direction, (LNNC) trivially holds (see the condition (R-8.1)).

In the sequel, we say that an attainable allocation $f$ is a coalitional equilibrium allocation if there exists a vector $p \in S_{+}^{\prime} \backslash\{0\}$ such that, for every coalition $E$ :

$$
\left\langle p, \int_{E} f \mathrm{~d} \mu\right\rangle=\left\langle p, \int_{E} e \mathrm{~d} \mu\right\rangle
$$

and

$$
x \in \int_{E} U_{f} \mathrm{~d} \mu \text { implies }\langle p, x\rangle>\left\langle p, \int_{E} e \mathrm{~d} \mu\right\rangle .
$$

We say that an attainable allocation $f$ is a Walrasian allocation if there exists a vector $p \in S_{+}^{\prime} \backslash\{0\}$ such that a.e. on $T$, $\langle p, f(t)\rangle=\langle p, e(t)\rangle$ and

$$
x \succ_{t} f(t) \text { implies }\langle p, x\rangle>\langle p, e(t)\rangle .
$$

The next theorem establishes the equivalence between the core and the set of coalitional equilibrium allocations.

Theorem 2. Let $\xi$ be an economy that satisfies assumptions (A0), (A1), (A3), (P1), (P2) and (LNNC). Then, an allocation belongs to $\mathcal{C}(\xi)$ if and only if it is a coalitional equilibrium allocation.

Proof. Since the "if" part is trivial, it suffices to prove the "only if" part. Let $f$ be a core allocation. Using Uhl's (1969) theorem one can easily modify the proof of Proposition 5 in Hildenbrand (1974, p. 62) to show that the set $C:=\mathrm{cl}\left[\bigcup\left\{\int_{E} U_{f}-\int_{E} e: E \in \Sigma, \mu(E)>0\right\}\right]$ is convex. Since int $S_{+}$is open, $C \cap-\operatorname{int} S_{+}=\emptyset$. Otherwise, the set $\int_{E} U_{f}-\int_{E} e$ would intersect - int $S_{+}$for a coalition $E$, and this would contradict the hypothesis that $f$ is a core allocation. First, assume $C$ is non-empty. Then, since -int $S_{+}$is an open convex cone, by a separating hyperplane theorem (see Dunford and Schwartz, 1967, Theorem V.2.8), there is a $p \in S_{+}^{\prime} \backslash\{0\}$ such that, for every coalition $E$ :

$$
x \in \int_{E} U_{f} \text { implies }\langle p, x\rangle \geq\left\langle p, \int_{E} e\right\rangle .
$$

Suppose now that there exist a coalition $E$ and an integrable function $g: E \rightarrow S$ with $\left\langle p, \int_{E}(g-e)\right\rangle=0$ such that $g(t) \succ_{t} f(t)$ a.e. on $E$. By Corollary 1, there exist a number $\alpha>0$ and a subcoalition $F$ of $E$ such that the point $x:=\int_{E} g+\alpha \int_{F}(\varphi-g)$ belongs to $\int_{E} U_{f}$, where $\varphi$ is as in the survival assumption (A3). By (3), we must have $\left\langle p, \int_{F}(g-e)\right\rangle \geq 0$, and hence, $\langle p, x\rangle=\left\langle p, \int_{E} e+\alpha \int_{F}(\varphi-g)\right\rangle \leq\left\langle p, \int_{E} e+\alpha \int_{F}(\varphi-e)\right\rangle$. Now, since $\alpha \int_{F}(\varphi-e)$ belongs to - int $S_{+}$, it follows that $\langle p, x\rangle<\left\langle p, \int_{E} e\right\rangle$, where we use the fact that $\langle p, u\rangle>0$ for all $u \in$ int $S_{+}$. This contradicts (3) and proves (2).

Notice that from the assumption (LNNC) and (2) it immediately follows that $\left\langle p, \int_{E} f\right\rangle \geq\left\langle p, \int_{E} e\right\rangle$ for any coalition $E$ with $\int_{E} U_{f} \neq \emptyset$. Suppose now there exists a coalition $E$ such that $\langle p, f(t)\rangle\langle\langle p, e(t)\rangle$ for a.e. $t \in E$. This implies $\left\langle p, \int_{F} f\right\rangle\left\langle\left\langle p, \int_{F} e\right\rangle\right.$ for any subcoalition $F$ of $E$. In particular, $\left\langle p, \int_{E} f\right\rangle\left\langle\left\langle p, \int_{E} e\right\rangle\right.$, and hence, $\int_{E} U_{f}=\emptyset$. From (LNNC) it then follows that there exists a subcoalition $E_{0}$ of $E$ such that $\int_{E_{0}} f \geq \int_{E_{0}} e$. But then $\left\langle p, \int_{E_{0}} f\right\rangle \geq$ $\left\langle p, \int_{E_{0}} e\right\rangle$, a contradiction. This shows that $\langle p, f(t)\rangle \geq\langle p, e(t)\rangle$ a.e. on $T$. Since $f$ is attainable and $p$ is positive, we conclude that $\langle p, f(t)\rangle=\langle p, e(t)\rangle$ a.e. on $T$. This completes the proof for the case $C \neq \emptyset$.

Finally, suppose $C=\emptyset$ and take any $p \in S_{+}^{\prime} \backslash\{0\}$. Note that for every coalition $E$ the set $\int_{E} U_{f}$ is empty and the statement (2) is voidly true. Moreover, by (LNNC), every coalition $E$ has a subcoalition $E_{0}$ with $\int_{E_{0}} f \geq \int_{E_{0}} e$. Applying the argument in the preceding paragraph, we see that $\langle p, f(t)\rangle=\langle p, e(t)\rangle$ a.e. on $T$.

Remark 11. Proof of Theorem 2 shows that when the conclusion of Corollary 1 holds, every coalitional quasiequilibrium is a coalitional equilibrium, that is, conditions (1) and (3) hold if and only if conditions (1) and (2) hold.

The trouble is $\int_{E} U_{f} \mathrm{~d} \mu$ may be empty even if $U_{f}(t)$ is non-empty a.e. on $E$. 
In the next result, we apply Theorem 2 to give a proof of Podczeck's (2003) assertion: Given any core allocation in an atomless economy, there exists a price system which makes this allocation a Walrasian allocation in every separable subeconomy. In view of Remark 10 and the discussion in Section 3.1, this result is a technical extension of core-Walras equivalence theorems of Rustichini and Yannelis (1991, Theorem 4.1) and Podczeck (2003, Theorem 4) to the case of a non-separable commodity space. ${ }^{22}$

Corollary 4. Let $\xi$ be an economy that satisfies assumptions (A0), (A1), (A3), (P1), (P2) and (LNNC). Then, an attainable allocation $f$ belongs to $\mathcal{C}(\xi)$ if and only if there exists a $p \in S_{+}^{\prime} \backslash\{0\}$ such that $\langle p, f(t)\rangle=\langle p$,e $e(t)\rangle$ a.e. on $T$, and for every separable subset $Q$ of $S$ :

$$
\text { a.e. on } T: \quad x \in Q \text { and } x \succ_{t} f(t) \quad \text { imply } \quad\langle p, x\rangle>\langle p, e(t)\rangle .
$$

Proof. Since integrable functions are essentially separably valued, the "if" part is obvious. To prove the "only if" part, let $f$ be a core allocation. Apply Theorem 2 and obtain a $p \in S_{+}^{\prime} \backslash\{0\}$ with $\langle p, f(t)\rangle=\langle p, e(t)\rangle$ a.e. on $T$ that satisfies (2). Let $Q \subset S$ be separable, and ignoring a null set, let $Y$ be a separable, closed, linear subspace such that $Q \cup f(T) \subset Y$. For every $t \in T$ define $\Psi(t):=\{x \in Y:\langle p, x-e(t)\rangle \leq 0\} \cap U_{f}(t)$. Notice that by the measurability assumption (P1), $\mathrm{Gr}_{\Psi}$ belongs to $\Sigma \otimes \mathfrak{B}(Y)$. Hence, clearly, the set $E:=\{t \in T: \Psi(t) \neq \emptyset\}$ is measurable. Obviously, to complete the proof it suffices to show that $\mu(E)=0$. Suppose to the contrary $\mu(E)>0$. By Aumann's (1969) measurable selection theorem, there exists a measurable function $g: E \rightarrow Y$ such that $g(t) \in \Psi(t)$ a.e. on $E$. For each $n \in \mathbb{N}$, let $g_{n}: E \rightarrow Y$ be a simple function such that $\lim _{n} g_{n}(t)=g(t)$ a.e. on $E$. Now, by Egoroff theorem (see Dunford and Schwartz, 1967, Theorem III.6.12), there exists a subcoalition $E_{0}$ of $E$ such that $g_{n}(t)$ converges to $g(t)$ uniformly on $E_{0}$. But then $g$ must be integrable over $E_{0}$. By construction, this contradicts (2).

An obvious fact, also implied by the results above, is that every Walrasian allocation is a coalitional equilibrium allocation. On the other hand, in view of the examples of Tourky and Yannelis (2001) and Podczeck (2003), the converse is not true. Proof of Corollary 4 demonstrates a way of understanding the nature of the problem: Even if it is non-empty valued on every member of a coalition $E$, the correspondence $\Psi(t) \equiv\{x \in Y:\langle p, x-e(t)\rangle \leq 0\} \cap U_{f}(t)$ may not admit a measurable selection over $E$, provided that $Y$ is non-separable. This, in turn, may prevent the existence of a subcoalition $E_{0}$ of $E$ for which the aggregate alternative $\int_{E_{0}} f \mathrm{~d} \mu$ is suboptimal at the given price vector $p$. In other words, it may not be possible to transform the individual alternatives, which are affordable at given prices, and which are preferred to a given allocation, into an aggregate preferred alternative, no matter over which subcoalition we try to carry out this aggregation procedure.

The next result presents two useful implications of Theorem 2: A core allocation in an atomless economy is stable in the sense of Cornwall (1969) and belongs to the strong core.

Corollary 5. Let $\xi:=\{(T, \Sigma, \mu), S, X, e, \succ\}$ be an economy that satisfies assumptions (A0), (A1), (A3), (P1), (P2) and (LNNC), and let $f$ be a core allocation of $\xi$.

(a) Then, f belongs to the core of the economy $\xi^{\prime}:=\{(T, \Sigma, \mu), S, X, f, \succ\}$.

(b) If $\xi$ also satisfies assumption (P4), then f belongs to $\mathcal{S C}(\xi)$.

Proof. Let $p$ be a vector in $S_{+}^{\prime} \backslash\{0\}$ that satisfies (1) and (2). Then, for any coalition $E$ and any $x \in \int_{E} U_{f},\langle p, x\rangle>$ $\left\langle p, \int_{E} e\right\rangle=\left\langle p, \int_{E} f\right\rangle$. Since $p$ is positive, we cannot have $x \leq \int_{E} f$. This proves part (a). To prove part (b), let $E$ be a coalition and suppose that $g: E \rightarrow S$ is an integrable function such that $g(t) \succsim_{t} f(t)$ a.e. on $E$ and $g(t) \succ_{t} f(t)$ a.e. on some subcoalition $E_{0}$ of $E$. Suppose $\langle p, g(t)\rangle<\langle p, e(t)\rangle$ for all $t$ in a measurable set $B \subset E \backslash E_{0}$ with $\mu(B)>0$. By (LNNC), this implies $\int_{B} U_{g} \neq \emptyset$, and hence, $\int_{B} g \in \operatorname{cl} \int_{B} U_{g}$. Note that, by (P4), $\int_{B} U_{g} \subset \int_{B} U_{f}$. Thus, from (2) it follows that $\left\langle p, \int_{B} g\right\rangle \geq\left\langle p, \int_{B} e\right\rangle$, a contradiction. Hence, $\langle p, g(t)\rangle \geq\langle p, e(t)\rangle$ a.e. on $E \backslash E_{0}$. Combined with (2), this implies $\left\langle p, \int_{E} g\right\rangle>\left\langle p, \int_{E} e\right\rangle$. Since $p$ is positive, we cannot have $\int_{E} g \leq \int_{E} e$.

\footnotetext{
22 The measurability and survival assumptions of Rustichini and Yannelis (1991) are weaker than those used here. They also avoid free disposal. On the other hand, they make the additional assumption that preferences are monotone and ordered.
} 
Remark 12. In view of Corollary 5(b), if preferences are ordered, under the hypotheses of Theorem 2, the strong core and the set of coalitional equilibrium allocations coincide.

\subsection{Continuity of the core correspondence}

In this section, we present an infinite dimensional extension of Kannai's (1970) theorem on the continuity of the core correspondence. In the sequel, we endow $S \times S$ with a norm which generates the product topology. Since we identify preference relations of agents as subsets of $S \times S$, the Hausdorff distance between two preference relations is defined via this norm as in Section 2.

Theorem 3. Suppose that the commodity space $S$ and the consumer space $(T, \Sigma, \mu)$ satisfy (A0). Let $\xi:=\{(T, \Sigma, \mu), S, X, e, \succ\}$ be an economy and take a sequence $\xi_{n}:=\left\{(T, \Sigma, \mu), S, X_{n}, e_{n}, \succ^{n}\right\}(n \in \mathbb{N})$ that converges to $\xi$ in the sense that $\lim _{n} \int_{T}\left\|e-e_{n}\right\| \mathrm{d} \mu=\lim _{n} \sigma\left(\succsim_{t}, \succsim_{t}^{n}\right)=0$ a.e. on T. Assume further that $\xi$ satisfies assumptions (A1)-(A3), (P1)-(P4), and $\xi_{n}$ satisfies assumptions $(\mathrm{P} 1)$ and $(\mathrm{P} 4)$ for every $n \in \mathbb{N}$. Let $\left\{f_{n}\right\}$ be a sequence of functions such that $f_{n} \in \mathcal{C}\left(\xi_{n}\right)$ for all $n \in \mathbb{N}$, and suppose further that:

(i) There exists an integrable function $q: T \rightarrow \mathbb{R}$ such that $\sup _{n}\left\|f_{n}(t)\right\| \leq q(t)$ a.e. on $T$.

(ii) $w-\lim _{n} f_{n}(t)$ exists a.e. on $T$.

Then, under any of the following two conditions, the function defined by $f(t):=w-\lim _{n} f_{n}(t)(t \in T)$ is a core allocation in the economy $\xi$.

(a) $X_{n}=X$ for all $n \in \mathbb{N}$.

(b) $S$ is separable and $\mathrm{Gr}_{X_{n}} \in \Sigma \otimes \mathfrak{B}(S)$ for all $n \in \mathbb{N} .^{23}$

Remark 13. As we shall see shortly, in the proof of Theorem 3, given any allocation $g$ in the economy $\xi$, we need to find an allocation $g_{n}$ in the economy $\xi_{n}(n \in \mathbb{N})$ such that the sequence $\left\{g_{n}\right\}$ converges to $g$ pointwisely. If the commodity space is non-separable, convergence of preferences in the Hausdorff distance may not be sufficient for this purpose, for the consumption sets in the approximating economies can be too dispersed across consumers. Hence the need for conditions (a) or (b). We also emphasize that even if it allows non-separability, condition (a) is not innocuous: If the limit economy is being approximated by a sequence of economies obtained from a discretization of the set of consumers (see Martins-da-Rocha, 2003; Araujo et al., 2004), then unless consumption sets across agents are constant, consumption set correspondences across economies would not be constant.

It is worth to note that in contrast to Kannai's (1970) original approach, in Theorem 3 we do not assume non-atomicity. This, in turn, prevents the exploitation of the price characterization of core allocations on separable subeconomies. Following Grodal's (1971) approach, we use here the conclusion of Lemma 1 to show directly that the limit of a sequence of core allocations is in the core. On the other hand, this approach necessitates the admittedly strong pointwise convergence condition (ii). We note, however, that if the sequence $\left\{\xi_{n}\right\}$ consists of atomless economies that satisfy hypotheses of Corollary 4, and if the preferences in the economy $\xi$ are convex, under a set of further mild assumptions, one can replace this condition with a Fatou-type convergence condition " $f(t) \in \overline{\mathrm{co}} w-L s_{n} f_{n}(t)$ a.e. on $T ., 24$ We close the discussion with a proof of Theorem 3.

Proof of Theorem 3. Since $\succsim_{t}^{n}$ is reflexive for all $t \in T$ and all $n \in \mathbb{N}$, whenever $\lim _{n} \sigma\left(\succsim_{t}, \succsim_{t}^{n}\right)=0$, there is a sequence $\left\{x_{n}^{t}\right\}$ in $X(t)$ such that $\lim _{n}\left\|x_{n}^{t}-f_{n}(t)\right\|=0$. Then, $f(t)=w-\lim _{n} x_{n}^{t}$ a.e. on $T$. Since $X(t)$ is closed and convex, it is weakly closed, and therefore, $f(t) \in X(t)$ a.e. on $T$. Note that for every $p \in S^{\prime}$ and every $n$, $\left|\left\langle p, f_{n}(t)\right\rangle\right| \leq q(t)\|p\|$ a.e. on $T$, and hence, by Lebesgue dominated convergence theorem, the definition of $f$ implies

\footnotetext{
${ }^{23}$ For various alternative continuity results for the case of a differential information economy with finitely many consumers and infinitely many states, see Einy et al. (2005) and Balder and Yannelis (2006).

${ }^{24}$ This assertion can be proved by applying Corollary 4 to each member of the sequence $\left\{\xi_{n}\right\}$ and then by following similar arguments to those of, for instance, Martins-da-Rocha (2003, Claim 5.1) or Araujo et al. (2004, Claims 5.2 and 5.4). Similarly, one can show that in an atomless economy that satisfies the hypotheses of Podczeck's (2003) core non-emptiness result Theorem 2, the core is weakly compact in $L_{1}(\mu, S)$.
} 
$\lim _{n} \int_{T}\left\langle p, f_{n}(t)\right\rangle=\int_{T}\langle p, f(t)\rangle$. Moreover, for a.e. $t \in T, f(t)$ belongs to $\overline{\operatorname{co}}\left\{f_{n}(t): n \in \mathbb{N}\right\}$, and thus, $f$ is essentially separably valued and $\|f(t)\| \leq q(t)$ a.e. on $T$. So, $f$ is integrable (see Dunford and Schwartz, 1967, Theorems III.2.22 and III.6.11), and hence, for every $p \in S^{\prime}, \lim _{n}\left\langle p, \int_{T} f_{n}\right\rangle=\lim _{n} \int_{T}\left\langle p, f_{n}(t)\right\rangle=\int_{T}\langle p, f(t)\rangle=\left\langle p, \int_{T} f\right\rangle$ (see Dunford and Schwartz, 1967, Theorem III.2.19), that is, $\int_{T} f=w-\lim _{n} \int_{T} f_{n}$. Finally, note that since $S_{+}$is weakly closed, and since $\int_{T} f_{n} \leq \int_{T} e_{n}(n \in \mathbb{N})$, we have $\int_{T} f \leq \int_{T} e$. Thus, $f$ is an attainable allocation in the economy $\xi$.

Suppose now $f \notin \mathcal{C}(\xi)$ and let $E$ be a coalition that blocks $f$ via a function $g: E \rightarrow S$. By Lemma 1, we can assume $z:=\int_{E}(e-g) \in \operatorname{int} S_{+}$. We claim and later prove that under the conditions (a) or (b), for each $n \in \mathbb{N}$, there exists a measurable function $g_{n}: T \rightarrow S$ such that

$$
\lim _{n} g_{n}(t)=g(t) \quad \text { and } \quad g_{n}(t) \in X_{n}(t) \quad \text { for a.e. } t \in T .
$$

For each $m \in \mathbb{N}$, put $E_{m}:=\left\{t \in E: g_{n}(t) \succ_{t}^{n} f_{n}(t), \forall n \geq m\right\}$. Note that for each $m, E_{m} \subset E_{m+1}$, and since the economy $\xi^{n}$ satisfies the measurability assumption (P1) $(n \in \mathbb{N}), E_{m}$ belongs to $\Sigma$. For now let us assume that

$$
\bigcup_{m \in \mathbb{N}} E_{m} \sim E \text {. }
$$

Assuming (4) and (5) we can proceed as follows. By Egoroff theorem (see Dunford and Schwartz, 1967, Theorem III.6.12), for each $m$ there exists a measurable set $A_{m} \subset E_{m}$ with $\mu\left(E_{m} \backslash A_{m}\right)<1 / m$ such that as $n$ goes to infinity, $g_{n}(t)$ converges to $g(t)$ uniformly on $A_{m}$. Hence, there exists an increasing function $m \rightarrow k_{m}$ from $\mathbb{N}$ into $\mathbb{N}$ such that $k_{m} \geq m$ and $\int_{A_{m}}\left\|g_{k_{m}}-g\right\| \leq 1 / m$ for all $m$. Since $\lim _{m} \mu\left(E \backslash A_{m}\right)=\lim _{m} \mu\left(E \backslash E_{m}\right)=0$, from absolute continuity of integral it follows that $\lim _{m} \int_{A_{m}} g_{k_{m}}=\lim _{m} \int_{A_{m}} g=\int_{E} g$ and $\lim _{m} \int_{A_{m}} e_{k_{m}}=\lim _{m} \int_{A_{m}} e=\int_{E} e$, where in the last set of equalities we also use the assumption that $\lim _{m} \int_{T}\left\|e_{k_{m}}-e\right\|=0$. But then $\lim _{m} \int_{A_{m}}\left(e_{k_{m}}-g_{k_{m}}\right)=z$, and for a sufficiently large $m, \int_{A_{m}}\left(e_{k_{m}}-g_{k_{m}}\right)$ belongs to int $S_{+}$. This, in particular, implies $\mu\left(A_{m}\right)>0$. Finally, note that, by construction, $g_{k_{m}}(t) \succ_{t}^{k_{m}} f_{k_{m}}(t)$ for all $t \in A_{m}$. Hence, $A_{m}$ blocks $f_{k_{m}}$ via $g_{k_{m}}$ in the economy $\xi_{k_{m}}$. This contradicts the hypothesis that $f_{k_{m}} \in \mathcal{C}\left(\xi_{k_{m}}\right)$ and proves that $f$ is in the core of $\xi$.

We next prove (5). Fix a point $t$ in $E$ with $g(t) \succ_{t} f(t)$ and assume that all pointwise convergence conditions hold at $t$. Now, by continuity assumptions (P2) and (P3) and by the assumption of ordered preferences (P4), there exist a norm open neighborhood $U$ of $g(t)$ and a weakly open neighborhood $W$ of $f(t)$ such that for all $x, y \in X(t),(x, y) \in U \times W$ implies $x \succ_{t} y$. Now, if $t$ does not belong to $\bigcup_{m \in \mathbb{N}} E_{m}$, we can find an increasing function $j \rightarrow n_{j}$ from $\mathbb{N}$ into $\mathbb{N}$ such that $f_{n_{j}}(t) \succsim_{t}^{n_{j}} g_{n_{j}}(t)$ for all $j \in \mathbb{N}$. Since $\lim _{j} \operatorname{dist}\left(\left(f_{n_{j}}(t), g_{n_{j}}(t)\right), \succsim_{t}\right)=0$, there exists a sequence $\left\{\left(y_{j}, x_{j}\right)\right\}$ in $\succsim_{t}$ such that $\lim _{j}\left\|y_{j}-f_{n_{j}}(t)\right\|=\lim _{j}\left\|x_{j}-g_{n_{j}}(t)\right\|=0$. But then, $w-\lim _{j} y_{j}=w-\lim _{j} f_{n_{j}}(t)=f(t)$ and $\lim _{j} x_{j}=$ $\lim _{j} g_{n_{j}}(t)=g(t)$. Thus, for a sufficiently large $j$ we must have $x_{j} \succ_{t} y_{j}$. This contradicts the supposition that $y_{j} \succsim_{t} x_{j}$. Hence, as we claimed, $t$ belongs to $E_{m}$ for a sufficiently large $m$.

We finally prove that there exists a sequence of functions $\left\{g_{n}\right\}$ from $T$ into $S$ which satisfy (4). First note that if $X_{n}=X$ for all $n$, we can simply let $g_{n}=g$ for all $n$. Now suppose that the condition (b) holds. Since $\succsim_{t}$ is reflexive

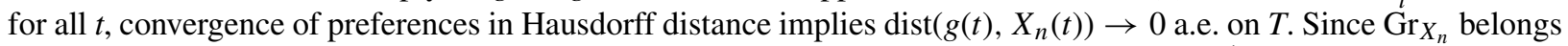
to $\Sigma \otimes \mathfrak{B}(S)$, and since $S$ is separable, the real function $t \rightarrow d_{n}(t):=\operatorname{dist}\left(g(t), X_{n}(t)\right)+n^{-1}$ is measurable for all $n$. Hence, the graph of the correspondence $\Psi_{n}: t \Rightarrow X_{n}(t) \cap B_{d_{n}(t)}(g(t))$ belongs to $\Sigma \otimes \mathfrak{B}(S)(n \in \mathbb{N})$. Moreover, by definition of the distance function, $\Psi_{n}(t)$ is non-empty a.e. on $T$. Hence, by applying Aumann's (1969) measurable selection theorem to the correspondence $\Psi_{n}$ we obtain the desired function $g_{n}(n \in \mathbb{N})$.

\section{Acknowledgments}

We are grateful to three anonymous referees of Journal of Mathematical Economics for various helpful remarks, especially for calling our attention to several references which were vital for this paper. We owe special thanks to Nicholas C. Yannelis and the third referee for many detailed suggestions which improved the exposition significantly. We are fully responsible for all remaining errors and deficiencies.

\section{Appendix A. A differential information economy}

Here we construct a differential information economy in the sense of Radner (1968) which generalizes the model of Hervés-Beloso et al. (2005) in several dimensions and which satisfies all assumptions that we used in Sections 3.3 
and 3.4 so that our results on the blocking power of small or big coalitions and core-Walras equivalence can be applied to a framework with differential information.

As in the main body of the paper, $(T, \Sigma, \mu)$ denotes a finite, complete, positive measure space of consumers. $\Omega$ is a non-empty, finite set which describes states of the nature. The collection of events is given by an algebra $\mathcal{A}$ of subsets of $\Omega$. We say that a partition $\mathcal{P}$ of $\Omega$ is measurable if $\mathcal{P} \subset \mathcal{A}$. Trade, or the implementation of an allocation takes place before the realization of the state. Hence, the commodity space is defined as the set of all functions from $\Omega$ into an ordered Banach space $\mathcal{X}$, with int $\mathcal{X}_{+} \neq \emptyset$, denoted by $S:=\mathcal{X}^{\Omega}$. We equip $S$ with the pointwise algebraic operations, a product norm, and the product order so that it becomes an ordered Banach space with $S_{+}=\left(\mathcal{X}_{+}\right)^{\Omega}$ and int $S_{+}=\left(\text {int } \mathcal{X}_{+}\right)^{\Omega}$. Each agent $t$ is equipped with a random utility function $V_{t}: \Omega \times \mathcal{X}_{+} \rightarrow \mathbb{R}$, a random endowment $e(t) \in S_{+}$, a prior $\pi_{t}$ on $\Omega$, and a measurable partition $\mathcal{P}(t)$ of $\Omega$. Here, $\mathcal{P}(t)$ describes the private information of an agent $t$, i.e., the agent $t$ can discriminate between states which belong to different events in $\mathcal{P}(t)$, but cannot distinguish those states which belong to the same event in $\mathcal{P}(t)$. An agent $t$ evaluates a random consumption bundle $x \in S_{+}$with the associated expected utility $U(t, x):=\sum_{\omega \in \Omega} \pi_{t}(\omega) V_{t}(\omega, x(\omega))$.

As a logical compatibility requirement, consumption choices and the endowment of an agent must not bear any information which is not available to her. Hence, the consumption set of an agent $t$ is given by $X(t):=\left\{x \in S_{+}\right.$: $\left.\forall P \in \mathcal{P}(t), \forall \omega, \omega^{\prime} \in P, x(\omega)=x\left(\omega^{\prime}\right)\right\}$, and $e(t)$ is assumed to be an element of $X(t)$. It is clear that $X(t)$ is a closed, convex subset of $S_{+}$. This completes the description of our differential information economy which is again denoted by $\xi:=\{(T, \Sigma, \mu), S, X, e, \succ\}$, where $\succ$ denotes the preference correspondence induced by $U(\cdot, \cdot)$.

Before we proceed, let us note that for any $p \in S^{\prime}$ and any $\omega \in \Omega$ we can define a function $p_{\omega} \in \mathcal{X}^{\prime}$ by $\left\langle p_{\omega}, a\right\rangle:=\left\langle p, a^{\omega}\right\rangle$ where for every $a \in \mathcal{X}, a^{\omega} \in S$ is the random vector which assigns $a$ to the state $\omega$ and 0 to every other state, so that $\langle p, x\rangle=\sum_{\omega \in \Omega}\left\langle p_{\omega}, x(\omega)\right\rangle$ for every $x \in S$. Hence, instead of defining a price system as an element of $S_{+}^{\prime}$, we can equivalently view a price system as a vector $\left(p_{\omega}\right)_{\omega \in \Omega}$ where $p_{\omega} \in \mathcal{X}_{+}^{\prime}$ represents ex ante prices of commodities contingent to the state $\omega$. We also note that for the differential information economy $\xi$, the notion of a Walrasian allocation that we defined in Section 3 coincides with the definition of an equilibrium allocation introduced by Radner (1968), and the core, as defined in Section 3, coincides with the notion of private core introduced by Yannelis (1991b).

Let us now make the following assumptions.

(A.A1). The function $t \rightarrow \mathcal{P}(t)$ is measurable, that is, for every measurable partition $\mathcal{P}$ of $\Omega$, the set $T_{\mathcal{P}}:=\{t \in T$ : $\mathcal{P}(t)=\mathcal{P}\}$ belongs to $\Sigma$.

(A.A2). The function $t \rightarrow \pi_{t}$ is measurable, that is, for every $\omega \in \Omega$, the real function $t \rightarrow \pi_{t}(\omega)$ is measurable, or equivalently, for every Borel subset $B$ of the $|\Omega|-1$ dimensional unit simplex, the set $\left\{t \in T: \pi_{t} \in B\right\}$ belongs to $\Sigma$.

(A.A3). For every $\omega \in \Omega,(t, a) \rightarrow V_{t}(\omega, a)$ is a Carathéodory function on $T \times \mathcal{X}_{+}$, that is, for every $(\omega, t) \in \Omega \times T$ the real function $V_{t}(\omega, \cdot)$ is (norm) continuous on $\mathcal{X}_{+}$, and for every $(\omega, a) \in \Omega \times \mathcal{X}_{+}$, the real function $V_{(\cdot)}(\omega, a)$ is measurable on $T$.

(A.A4). For every $(\omega, t) \in \Omega \times T$, every $a \in \mathcal{X}_{+}$, and every $b \in \operatorname{int} \mathcal{X}_{+}, V_{t}(\omega, a+b)>V_{t}(\omega, a)$.

(A.A5). For every $(\omega, t) \in \Omega \times T, e(t)(\omega) \in \operatorname{int} \mathcal{X}_{+}$, or equivalently, for every $t \in T, e(t) \in \operatorname{int} S_{+}$.

Remark A. The monotonicity assumption (A.2) of Hervés-Beloso et al. (2005) is identical with (A.A4), and their survival assumption (A.4) is identical with (A.A5). They assume the agents have a common prior so that (A.A2) is trivially satisfied. Moreover, they assume that there is a measurable partition $\left\{I_{1}, \ldots, I_{n}\right\}$ of $T$ such that for each $i=1, \ldots, n$, agents in $I_{i}$ are identical with respect to all aspects. This implies that $t \rightarrow \mathcal{P}(t)$ is a simple function so that (A.A1) holds, and that $V_{(\cdot)}(\omega, a)$ is a simple function on $T$ for every $(\omega, a) \in \Omega \times \mathcal{X}_{+}$so that the measurability requirement in (A.A3) also holds. Finally, they assume $\mathcal{X}=l_{\infty}$ and $V_{t}(\omega, \cdot)$ is Mackey continuous on $\mathcal{X}_{+}$for every $(\omega, t) \in \Omega \times T$, which verifies the continuity requirement in (A.A3).

We shall finally verify that the economy $\xi$ satisfies the assumptions that we used in Sections 3.3 and 3.4. (A0) does not require a verification. Next note that $\operatorname{Gr}_{X}=\bigcup_{\mathcal{P}}\left(T_{\mathcal{P}} \times X_{\mathcal{P}}\right)$, where the union is taken over all measurable partitions $\mathcal{P}$ of $\Omega$, and $X_{\mathcal{P}}$ is the common consumption set of agents in $T_{\mathcal{P}}$. Hence, (A.A1) implies $\operatorname{Gr}_{X} \in \Sigma \otimes \mathfrak{B}\left(S_{+}\right)$, 
and this verifies (A1). Since $0 \in X(t)$ for every $t \in T$, (A.A5) immediately implies the survival assumption (A3) (see also Remark 5). In view of Remark 6, to verify (P1) it suffices to show that $U(\cdot, \cdot)$ is a Carathéodory function on $T \times S_{+}$. But this is an obvious consequence of (A.A2) and (A.A3) and the definition of $U(\cdot, \cdot)$. Moreover, continuity of $U(t, \cdot)$ for every $t$ implies the upper continuity condition (P2). Finally, to verify our local non-satiation conditions, take a point $b \in$ int $\mathcal{X}_{+}$and define the constant function $\tilde{b} \in \operatorname{int} S_{+}$by $\tilde{b}(\omega)=b$ for every $\omega \in \Omega$. Then for every $t \in T$, every $x \in X(t)$, and every $\gamma>0$, the point $x+\gamma \tilde{b}$ belongs to $X(t)$, and by (A.A4), $x+\gamma \tilde{b} \succ_{t} x$. This shows that the condition (R-8.1) is valid in the present model, and therefore, every allocation is coalitionwise locally non-satiating. This implies in particular that $\xi$ also satisfies (LNNC).

\section{References}

Aliprantis, C.D., Border, K.C., 1999. Infinite Dimensional Analysis. Springer, Berlin.

Araujo, A., Martins-da-Rocha, V.F., Monteiro, P.K., 2004. Equilibria in reflexive Banach lattices with a continuum of agents. Economic Theory 24 , 469-492.

Aumann, R.J., 1969. Measurable utility and the measurable choice theorem. In: Guilbaud, G.T. (Ed.), La Decision. Colloque Internationaux du C. N. R. S, Paris, pp. 15-26.

Balder, E.J., Yannelis, N.C., 2006. Continuity properties of the private core. Economic Theory 29, 453-464.

Cheng, H.H.C., 1991. The principle of equivalence. In: Khan, M.A., Yannelis, N.C. (Eds.), Equilibrium Theory in Infinite Dimensional Spaces. Springer-Verlag, New York, pp. 197-221.

Cornwall, R.R., 1969. The use of prices to characterize the core of an economy. Journal of Economic Theory 1, 353-373.

Cornwall, R.R., 1970. Convexity and continuity properties of preference functions. Zeitschrift für Nationalökonomie 30, 35-52.

Cornwall, R.R., 1972. Conditions for the graph and the integral of a correspondence to be open. Journal of Mathematical Analysis and Applications 39, 771-792.

Dunford, N., Schwartz, J.T., 1967. Linear Operators. Part I. Interscience, New York.

Einy, E., Moreno, D., Shitovitz, B., 2001. Competitive and core allocations in large economies with differential information. Economic Theory 18, 321-332.

Einy, E., Haimanko, O., Moreno, D., Shitovitz, B., 2005. On the continuity of equilibrium and core correspondences in economies with differential information. Economic Theory 26, 793-812.

Grodal, B., 1971. A theorem on correspondences and continuity of the core. In: Kuhn, H.W., Szegö, G.P. (Eds.), Differential Games and Related Topics. North-Holland, Amsterdam, pp. 221-233.

Grodal, B., 1972. A second remark on the core of an atomless economy. Econometrica 40, 581-583.

Hervés-Beloso, C., Moreno-García, E., Núnez-Sanz, C., Páscoa, M.R., 2000. Blocking efficacy of small coalitions in myopic economies. Journal of Economic Theory 93, 72-86.

Hervés-Beloso, C., Moreno-García, E., Yannelis, N.C., 2005. Characterization and incentive compatibility of Walrasian expectations equilibrium in infinite dimensional commodity spaces. Economic Theory 26, 361-381.

Hildenbrand, W., 1974. Core and Equilibria of a Large Economy. Princeton University Press, Princeton.

Hu, S., Papageorgiou, N.S., 1997. Handbook of Multivalued Analysis, vol. 1: Theory. Kluwer Academic Publishers, Dordrecht.

Hüsseinov, F., 2003. Theorems on correspondences and stability of the core. Economic Theory 22, 893-902.

Kannai, Y., 1970. Continuity properties of the core of a market. Econometrica 38, 791-815.

Khan, M.A., Yannelis, N.C., 1991. Equilibria in markets with a continuum of agents and commodities. In: Khan, M.A., Yannelis, N.C. (Eds.), Equilibrium Theory in Infinite Dimensional Spaces. Springer-Verlag, New York, pp. 233-248.

Liapounoff, A., 1940. On completely additive vector functions. Bulletin of the Academy of Sciences of the USSR 4, 465-478.

Martins-da-Rocha, V.F., 2003. Equilibria in large economies with a separable Banach commodity space and non-ordered preferences. Journal of Mathematical Economics 39, 863-889.

Podczeck, K., 2003. Core and Walrasian equilibria when agents' characteristics are extremely dispersed. Economic Theory 22, $699-725$.

Radner, R., 1968. Competitive equilibrium under uncertainty. Econometrica 36, 31-58.

Rustichini, A., Yannelis, N.C., 1991. Edgeworth's conjecture in economies with a continuum of agents and commodities. Journal of Mathematical Economics 20, 307-326.

Schmeidler, D., 1972. A remark on the core of an atomless economy. Econometrica 40, 579-580.

Sun, Y., Yannelis, N.C., 2007. Core, equilibria and incentives in large asymmetric information economies. Games and Economic Behavior 61, 131-155.

Tourky, R., Yannelis, N.C., 2001. Markets with many more agents than commodities: Aumann’s "hidden” assumption. Journal of Economic Theory 101, 189-221.

Uhl, J.J., 1969. The range of a vector valued measure. Proceedings of the American Mathematical Society 23, 158-163.

Vind, K., 1964. Edgeworth-allocations in an exchange economy with many traders. International Economic Review 5, $165-177$.

Vind, K., 1972. A third remark on the core of an atomless economy. Econometrica 40, 585-586.

Yannelis, N.C., 1991a. Integration of Banach-valued correspondences. In: Khan, M.A., Yannelis, N.C. (Eds.), Equilibrium Theory in Infinite Dimensional Spaces. Springer-Verlag, New York, pp. 2-35.

Yannelis, N.C., 1991b. The core of an economy with differential information. Economic Theory 1, 183-198. 